\title{
A Case of Mistaken Identity - An Unusual Presentation of Neoplastic Meningitis and a Reminder of the Hallmark Features
}

\author{
Catriona Davidson ${ }^{1}$, Katerina Achilleos ${ }^{2}$, Francesca Crawley $^{2}$, and William Petchey ${ }^{2}$ \\ ${ }^{1}$ Affiliation not available \\ ${ }^{2}$ West Suffolk Hospitals NHS Trust
}

June 22, 2020

\begin{abstract}
Neoplastic meningitis remains a diagnostic challenge due to its varied clinical manifestations. We discuss a presentation with dizziness with an initial diagnosis of Benign Paroxysmal Positional Vertigo. We highlight the hallmark findings and reflect on how clinicians may miss evolving features in recurrent attenders due to 'anchoring' to previous diagnoses.
\end{abstract}

\section{Key Clinical Message}

This case offers an opportunity for education on the manifestations of neoplastic meningitis, a revision of the hallmark investigative features, and a reminder of the utilisation of lumbar puncture in investigating unexplained neurological symptoms. Additionally, it emphasises the need for clinicians to avoid 'diagnostic anchoring' when faced with recurrent attenders.

\section{Introduction}

Neoplastic meningitis, also known as leptomeningeal carcinomatosis or carcinomatous meningitis, occurs due to metastatic spread to the arachnoid and/or pia mater. It is associated with both hematological and solid malignancies and its incidence is increasing. ${ }^{1}$ Whether this actually represents increasing recognition remains uncertain. The associated meningeal inflammation can lead to a wide variety of clinical presentations making diagnosis challenging. Previous case reports have detailed a range of clinical features including headache, altered mental status, and visual disturbances, often requiring repeated review prior to the eventual correct diagnosis of neoplastic meningitis being made. ${ }^{2-4}$ This case highlights another manifestation, namely dizziness, which was initially attributed to a diagnosis of Benign Paroxysmal Positional Vertigo (BPPV).

Although the prognosis of neoplastic meningitis is poor, early recognition and aggressive treatment can increase life expectancy. ${ }^{5}$ It is therefore important that clinicians are reminded of the varied presentations and the cardinal investigative findings that help make the diagnosis. Furthermore, this case hopes to serve as a reminder of the diagnostic challenge recurrent attenders present, and the risk of missing evolving symptoms due to fixation on initial diagnoses.

\section{Case Presentation}

\section{History/Examination}

A 57-year-old male presented to the Emergency Department recurrently over a 4-week period with dizziness, headache and subsequent nausea and vomiting. He had no relevant past medical or travel history. He worked as a traffic warden, was a smoker with a 30-pack year history and consumed minimal alcohol. He had been investigated thoroughly on his first two presentations, having undergone a CT and MRI Head (Figure 1 and 2) and admission with assessment by a consultant neurologist. With a history descriptive of positional 
vertigo, torsional nystagmus on left lateral gaze and a positive Semont Diagnostic Manoeuvre (and resolution with Epley's manoeurvres), he had been diagnosed with BPPV.

Following his diagnosis with BPPV he presented two further times to the Emergency Department, being admitted on the fourth presentation due to significant dehydration and progressive weight loss, ascribed to the nausea and vomiting. His blood tests on this occasion demonstrated a significant neutrophilia, a raised urea (in keeping with clinical dehydration), and an unexplained elevated Alkaline Phosphatase (317 U/L). He described no additional symptoms, abdominal examination was unremarkable and he did not display any new motor weakness. His diagnosis of BPPV was initially maintained until, on further exploration, he was found to now have features of cognitive impairment with an abbreviated mental test score of $6 / 10$, and an inability to follow three stage commands. Further investigations were subsequently arranged.

\section{Investigations}

A repeat MRI Head was performed (Figure 3) which demonstrated mild hydrocephalus with no clear cause. A lumbar puncture (LP) was conducted and analysis of the cerebral spinal fluid (CSF) showed an increased opening pressure, elevated white cell count (lymphocytic), high protein, extremely low glucose and a high lactate (Table 1). Viral PCR, microscopy, bacterial and fungal cultures all revealed no organisms. A CT scan of the Chest, Abdomen and Pelvis failed to identify a primary tumour, however it revealed widespread metastatic bony deposits suggesting the diagnosis of neoplastic meningitis (Figure 4).

\section{Outcome/Follow up}

After establishing the presumed diagnosis of neoplastic meningitis, a second high-volume lumbar puncture was performed for CSF cytology, and a bone biopsy was arranged. Sadly, the patient suddenly deteriorated and required palliation. He died four days later.

CSF cytology, returned post-mortem, demonstrated a poorly differentiated adenocarcinoma. Immunohistochemistry staining suggested this was of lung or upper gastrointestinal origin.

Discussion

Neoplastic meningitis continues to be diagnostically challenging due to its subtle, varied, and non-specific clinical features. This case illustrates an initial presentation of vertigo, with sub-acute progression to cerebral involvement and higher executive dysfunction. Headache remains the most common symptom of neoplastic meningitis (present in 66\% of cases) but is non-specific. $50 \%$ present with myelopathy, with symptoms such as lower limb weakness, paraesthesia or bowel or bladder disturbance. 35\% present with isolated or grouped cranial nerve palsies, and $15 \%$ present with cerebral dysfunction manifesting as higher cortical deficits such as confusion or dysphasia. ${ }^{5-6}$ In retrospect, it is likely that the initial dizziness in this case was related to an isolated $8^{\text {th }}$ (vestibulocochlear) nerve palsy with branch involvement leading to vestibular neuronitis.

Treatment of neoplastic meningitis is palliative and although cannot reverse existing neurological deficits may prevent deterioration. ${ }^{7}$ Aggressive treatment can increase survival time from 4-6 weeks to 6 months, ${ }^{5-7}$ highlighting the importance of early recognition and diagnosis. Clinicians must therefore be aware of the many manifestations and maintain a high index of suspicion.

With reference to diagnosis, a triad of factors including classical symptoms and signs, appropriate findings on MRI imaging, and CSF analysis should be used. ${ }^{5} \mathrm{MRI}$ imaging is used to look for features of meningeal enhancement, whilst CSF findings of increased lymphocytes, high protein, very low CSF:serum glucose ratio, and high lactate, illustrated perfectly in this case, are highly suggestive of a diagnosis. ${ }^{5-6}$ Positive cytology from CSF is gold standard although this cannot always be obtained and may require multiple, large volume LPs. ${ }^{5-6}$ This case exemplifies the important role an LP can play in investigating new neurological symptoms.

Lastly, this case provides us with the opportunity to reflect on reasons behind diagnostic error. The prevalence of, and harm, that can arise from diagnostic error is well documented in the literature ${ }^{8-9}$ and it is therefore important that, as clinicians, we reflect on our practice in order to understand the factors influencing our decision-making. This case offers a reminder of the particular challenge recurrent attenders 
present, and the bias that can arise from anchoring to an initial or previous diagnosis. This patient received a diagnosis of BPPV from a consultant neurologist and, despite changing clinical features on subsequent presentations, this diagnosis was maintained following review by three experienced general physicians. This highlights the importance of remaining open minded in such cases and recognising diagnostic bias that may exist.

List of Abbreviations

$\mathrm{BPPV}=$ Benign Paroxysmal Positional Vertigo

$\mathrm{CSF}=$ Cerebral Spinal Fluid

$\mathrm{CT}=$ Computed Tomography

$\mathrm{LP}=$ Lumbar puncture

MRI = Magnetic Resonance Imaging

Author Contributions

Author 1: Dr Catriona Davidson MBBS MRCP, CT2 Addenbrookes Hospital, catriona.davidson@nhs.net Joint lead author

Author 2: Dr Katerina Achilleos BSc (Hons) MBBS MRCP, Consultant Rheumatologist West Suffolk Hospital, katerina.achilleos@wsh.nhs.uk - Joint lead author

Author 3: Dr Francesca Crawley MBBCh, FRCP, Consultant Neurologist West Suffolk Hospital, francesca.crawley@wsh.nhs.uk - Author

Author 4: Dr William Petchey BSc (Hons) BM PhD MRCP, Consultant Nephrologist West Suffolk Hospital, william.petchey@wsh.nhs.uk - Senior author

References

1. Cancer Research UK. About Carcinomatous Meningitis 2014. https://www.cancerresearchuk.org/aboutcancer/secondary-cancer/carcinomatous-meningitis/about-carcinomatous-meningitis. Accessed August 202019.

2. Foo, C.T. Burrell L.M., Johnson, D.F. 2016. An unusual presentation of carcinomatous meningitis. Oxford Medical Case Reports 2016;8:omw068

3. Trivedi, T, Reddi, R, Agarwal, P, Arya, A. 2018. A case of carcinomatous meningitis with occult primary malignancy. Journal of Emergency and Critical Care Medicine 2018;2:21.

4. Little, J, Rajkumar, C, Saleem, W. 2019. A rare case of malignant meningitis from a likely bronchogenic primary cancer. Oxford Medical Case Reports 2019;1:omy114

5. Fields. M. 2013. How to Recognize and Treat Neoplastic Meningitis. Journal of the Advanced Practitioner in Oncology 4;3:155-160.

6. Chamberlain. M. 2008). Neoplastic Meningitis. The Oncologist 13;9:967-977

7. Pellerino, A, Bertero, L, Ruda, R, Soffietti, R. 2018. Neoplastic Meningitis in Solid Tumours: From diagnosis to personalised treatments. Therapeutic Advances in Neurological Disorders 2018;11:1756286418759618.

8. Scott, I. 2009. Errors in clinical reasoning: Causes and remedial strategies. BMJ Online June 2008;2:b1860

9. Graber, M.L. 2013. The incidence of diagnostic error in medicine. BMJ Quality and Safety 2013;22:ii21ii 27

10. Oxford Medical Education Lumbar Puncture CSF Interpretation

Figure Legends

1. Figure 1: Transverse slice of initial CT Head on first presentation. Normal scan

2. Figure 2: Transverse slice of initial MRI Head (T2 weighted) on first presentation. Normal scan 
3. Figure 3: Transverse slice of repeat MRI Head (T2 weighted) on fourth presentation showing new hydrocephalus

4. Figure 4: Sagittal Section of CT Scan post-CSF analysis, performed due to suspicion of neoplastic meningitis. Multiple vertebral metastases demonstrated.

5. Table 1: Table depicting the CSF analysis of the patient in this case

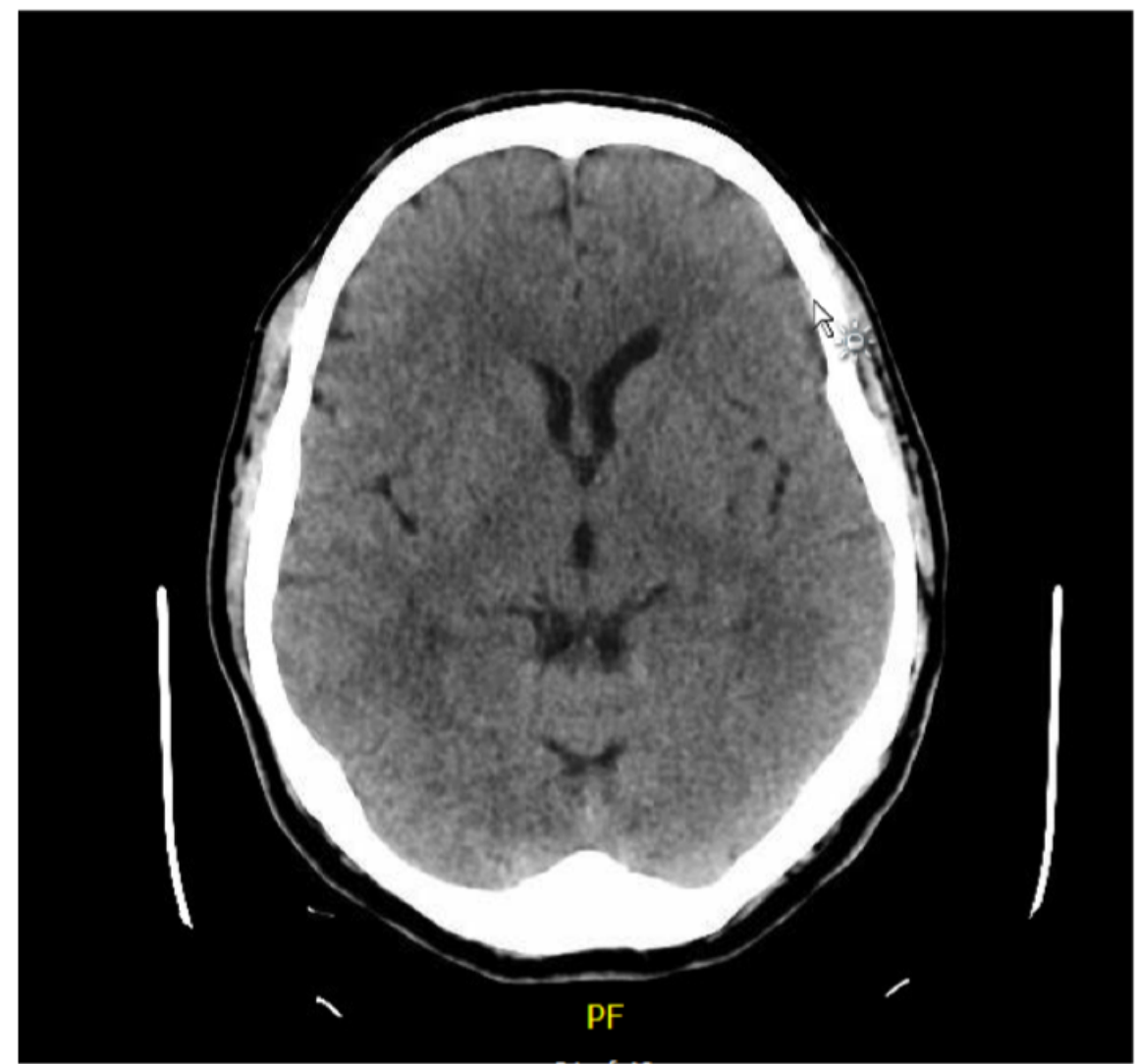




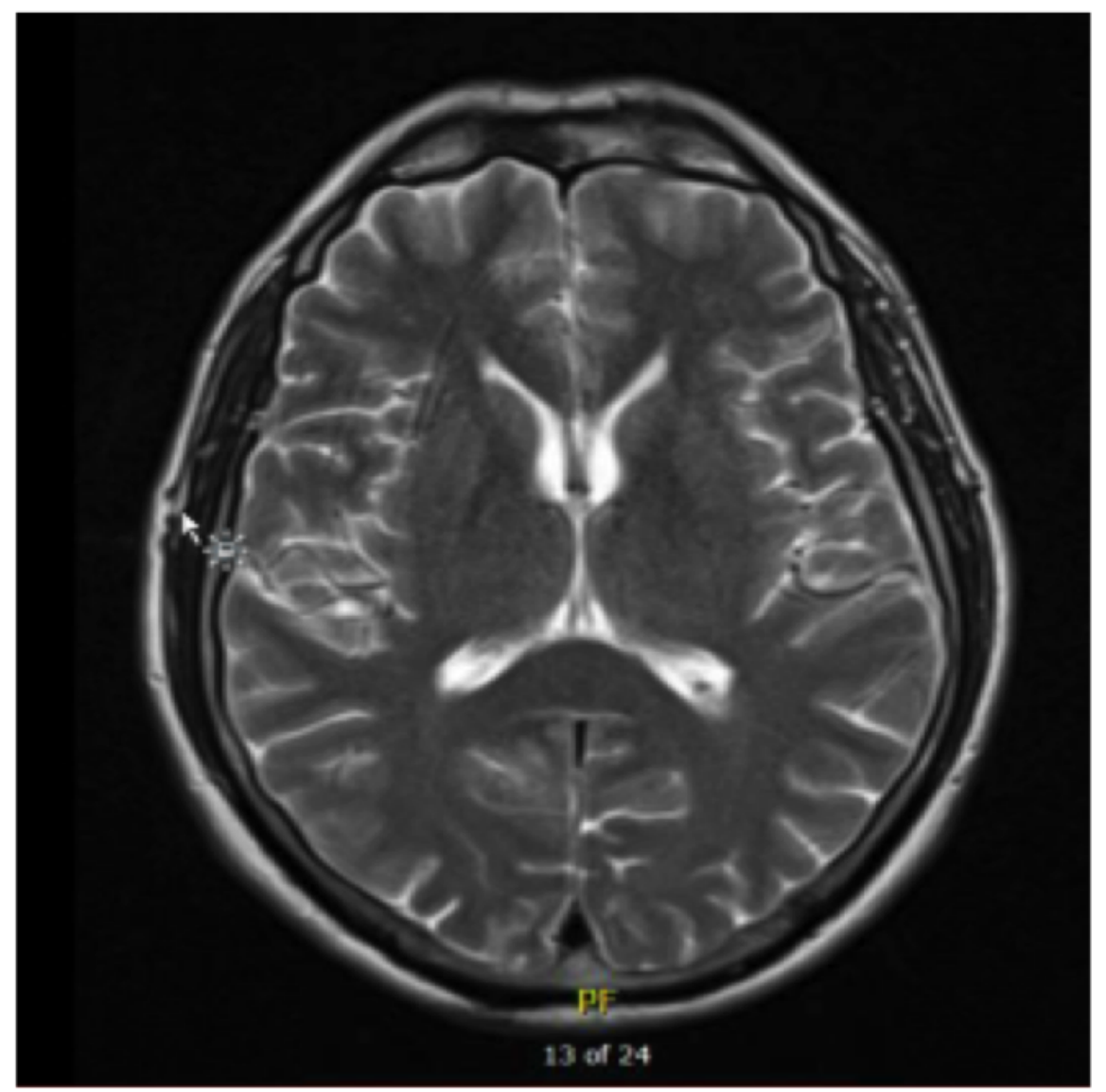




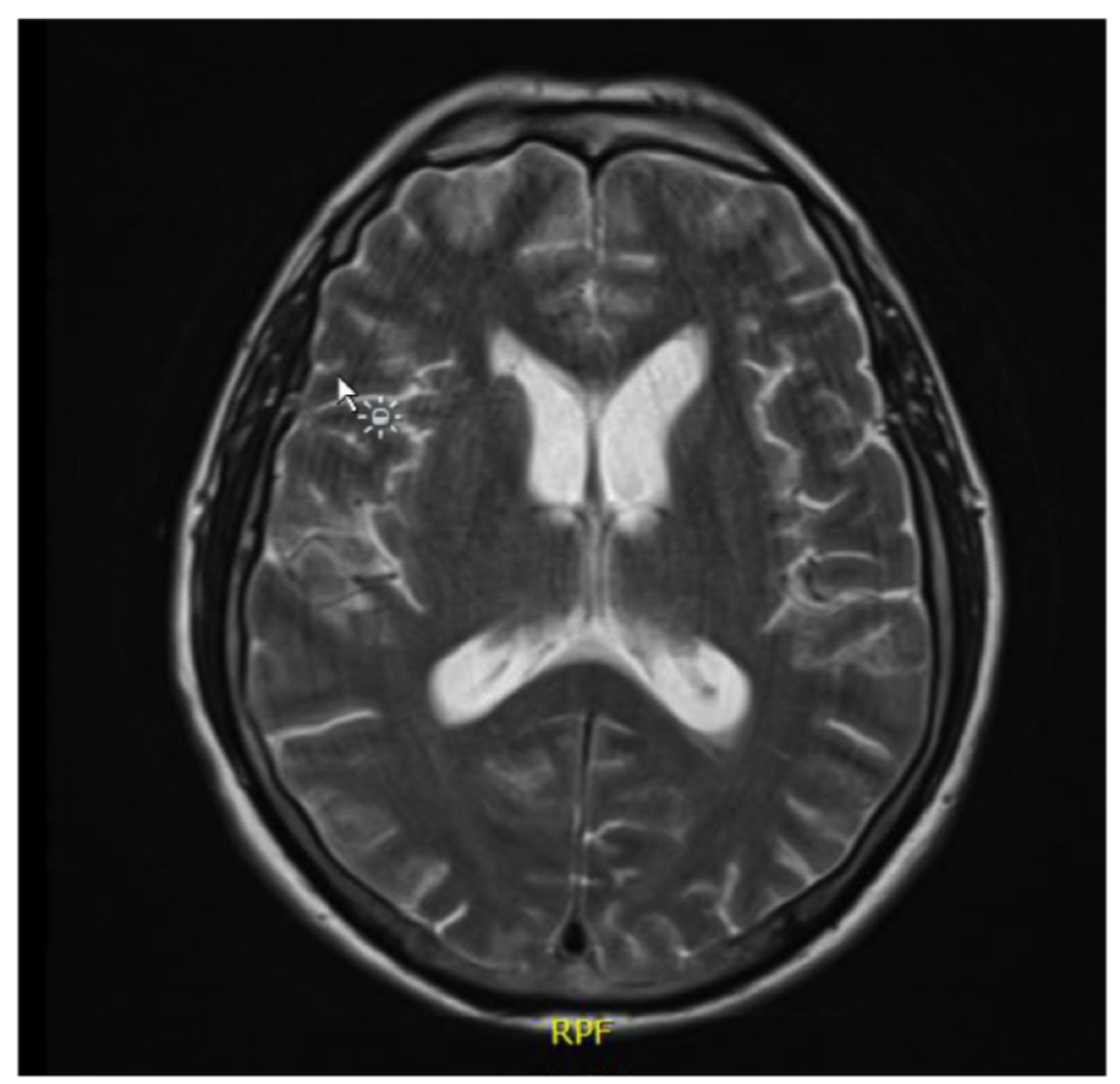




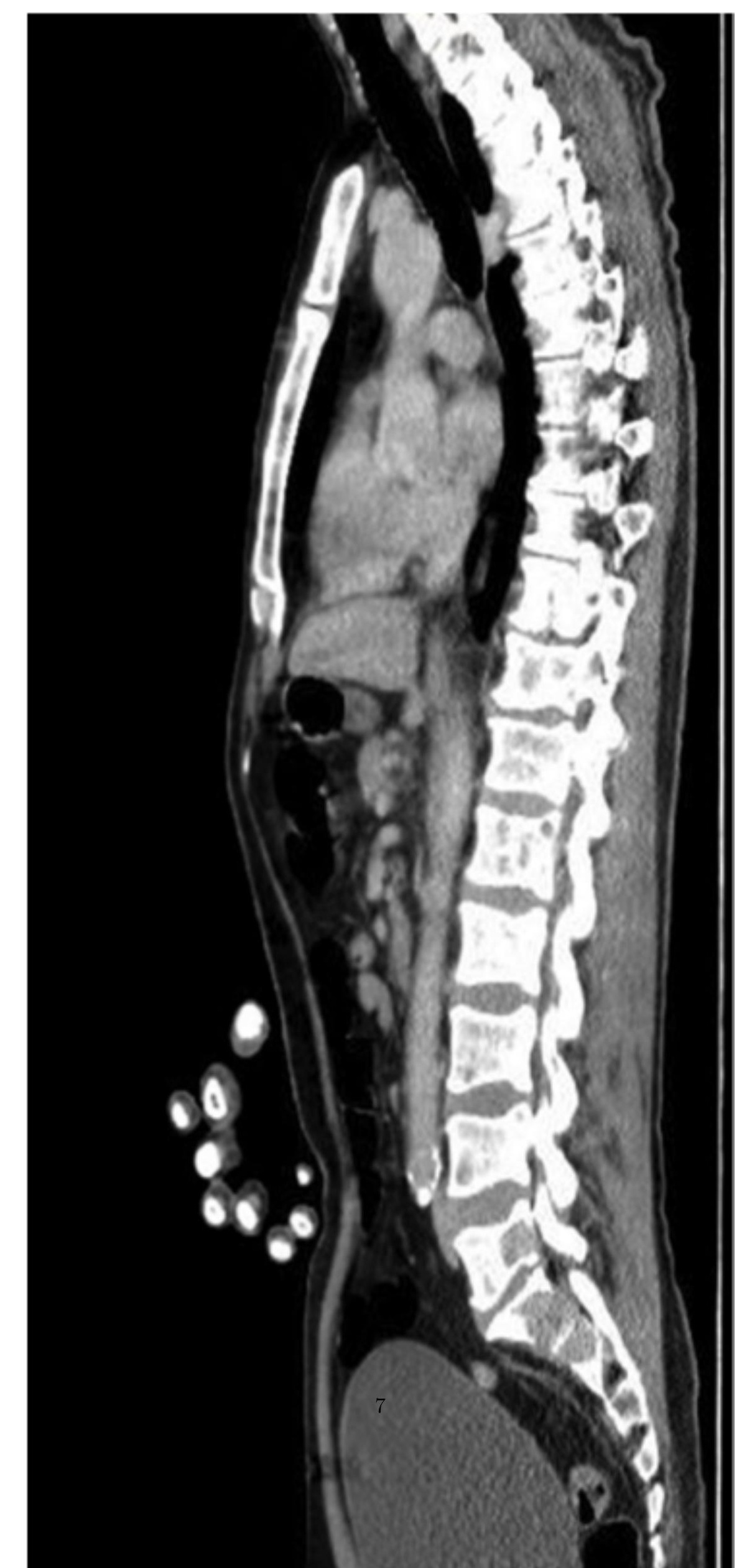




\begin{tabular}{|c|c|}
\hline CSF Results (normal values ${ }^{10}$ ) & Values \\
\hline Opening Pressure $\left(10-20 \mathrm{mmH}_{2} \mathrm{O}\right)$ & $34 \mathrm{mmH}_{2} \mathrm{O}$ \\
\hline White Cell Count ( $0-5$ cells $/ \mu \mathrm{L})$ & $\begin{array}{l}43 \times 10^{\wedge} 6 \\
100 \% \text { lymphocytes }\end{array}$ \\
\hline Protein $(<0.45 \mathrm{~g} / \mathrm{L})$ & $4.93 \mathrm{~g} / \mathrm{L}$ \\
\hline CSF Glucose ( $>60 \%$ serum glucose) & 0.7 \\
\hline Serum Glucose & 6.5 \\
\hline Lactate & $9.9 \mathrm{mmol} / \mathrm{L}$ \\
\hline Oligoclonal bands & Negative \\
\hline $\begin{array}{l}\text { MC\&S, Viral PCR, 5D-fungal cultures, } \\
\text { AFB }\end{array}$ & Negative - no organisms seen \\
\hline Xanthochromia & Not detected \\
\hline
\end{tabular}

